\title{
Breaking the Barrier-PEGylated Recombinant Human Hyaluronidase (PEGPH20)—A New Therapeutic Approach to the Treatment of Pancreatic Ductal Adenocarcinoma
}

\author{
Andrew Hendifar ${ }^{1}$ and Andrea Bullock ${ }^{2}$ \\ 1. Department of Medicine, Cedars-Sinai Medical Center, Los Angeles, US; 2. Division of Hematology-Oncology, Beth Israel Deaconess Medical \\ Center, Boston, US
}

DOI: https://doi.org/10.17925/OHR.2017.13.02.107

$\mathrm{N}$ ew therapeutic approaches are urgently needed to improve survival for patients with metastatic pancreatic ductal adenocarcinoma (PDA). This carcinoma is characterized by a hyaluronan (HA)-rich desmoplastic stroma that raises tumor interstitial fluid pressure (IFP), which in turn compresses the vasculature and impedes access of anti-cancer therapies and immune cells to tumor sites. It is this biophysical barrier that is the target for PEGylated recombinant human hyaluronidase (PEGPH20; pegvorhyaluronidase alfa), which degrades HA polymers to tetra- and hexa-saccharides to remodel the tumor stroma. In preclinical models, PEGPH2O reduced IFP, and expanded tumor vasculature to improve perfusion, which increased access for innate immune cells, antibodies and therapeutic agents. The results of a phase Ib study have suggested benefits in overall survival and progression-free survival (PFS) for patients with tumors that accumulate HA (termed HA-High) treated with a combination of gemcitabine and PEGPH2O. A phase II study (HALO 109-202) demonstrated that HA could be a potential biomarker for identifying patients who may be most suitable for PEGPH20 treatment. HALO 109-202 showed positive outcomes for PFS especially in HA-High patients treated with PEGPH2O plus nab-paclitaxel and gemcitabine. A randomized, double-blind, phase III study (HALO 109-301) exploring the benefits of PEGPH2O in HA-High patients with PDA is ongoing. Other PEGPH2O-based combinations are being investigated in multiple stroma-rich cancers, including lung, gastric, and breast. PEGPH2O is the most advanced therapy targeting the tumor stroma and has the potential to form the therapeutic backbone for the treatment of stroma-rich tumors.

\section{Keywords}

Pancreatic ductal adenocarcinoma (PDA), tumor microenvironment (TME), hyaluronan (HA), PEGylated recombinant human hyaluronidase (PEGPH2O)

Disclosure: Andrew Hendifar has participated in a consulting or advisory role with Novartis, Ipsen, Perthera, and Xbiotech and received travel/accommodation/expenses from Halozyme. Andrea Bullock has stock and other ownership interests in Amgen, Johnson \& Johnson, and Medtronic, participated in a consulting or advisory role with Celgene, Halozyme, and Bayer, and received travel/accommodation/expenses from Halozyme

Acknowledgments: Medical writing assistance was provided by James Gilbart at Touch Medical Media, and funded by Halozyme.

Compliance with Ethics Guidelines: This study involves a review of the literature and did not involve any studies with human or animal subjects performed by any of the authors.

Authorship: All named authors meet the International Committee of Medical Journal Editors (ICMJE) criteria for authorship of this manuscript, take responsibility for the integrity of the work as a whole, and have given final approval to the version to be published.

open Access: This article is published under the Creative Commons Attribution Noncommercial License, which permits any noncommercial use, distribution, adaptation, and reproduction provided the original author(s) and source are given appropriate credit.

Received: September 29, 2017

Accepted: November 7, 2017

Citation: Oncology \& Hematology Review, 2017;13(2):107-11

Corresponding Author: Andrew Hendifar, Department of Medicine, Cedars-Sinai Medical Center, Los Angeles, CA 90048, US. E: Andrew.Hendifar@cshs.org

Support: The publication of this article was supported by Halozyme. The views and opinions expressed are those of the author and do not necessarily reflect those of Halozyme.
Despite progress in managing many cancer types in recent decades, metastatic pancreatic ductal adenocarcinoma (PDA), accounting for over $90 \%$ of all malignancies of the pancreas, continues to have an extremely poor prognosis. Indeed, in the US PDA has an estimated 5-year survival rate of $8 \%$ for all patients and $3 \%$ for metastatic disease. ${ }^{1,2}$ PDA is now the third leading cause of cancer death in the US and this is predicted to increase to be the second biggest cause of cancer death in the US by 2020-2030.3,4

Outcomes in PDA have not improved substantially over the last 30 years. ${ }^{1}$ This mainly arises from the patient having reached a metastatic stage at the time of diagnosis with poor response to therapy. The lack of progress in developing effective therapies for PDA is due to the nature of the disease: tumor cells co-opt multiple cellular and extracellular mechanisms to form a complex cancer organ with a marked propensity towards metastasis and resistance to therapy. ${ }^{5}$ Against this bleak picture, improved understanding of the pathophysiology behind PDA, the identification of molecular mechanisms underlying its aggressive nature, and improved access to the tumor site have enabled some exciting developments. This increased knowledge raises hope for more successful approaches to managing this disease.

\section{The tumor microenvironment}

The development, progression and aggressiveness of PDA are significantly affected by components of the tumor microenvironment (TME), which 
Figure 1: PEGylated recombinant human hyaluronidase (PEGPH20) targets hyaluronan (HA) in the tumor microenvironment

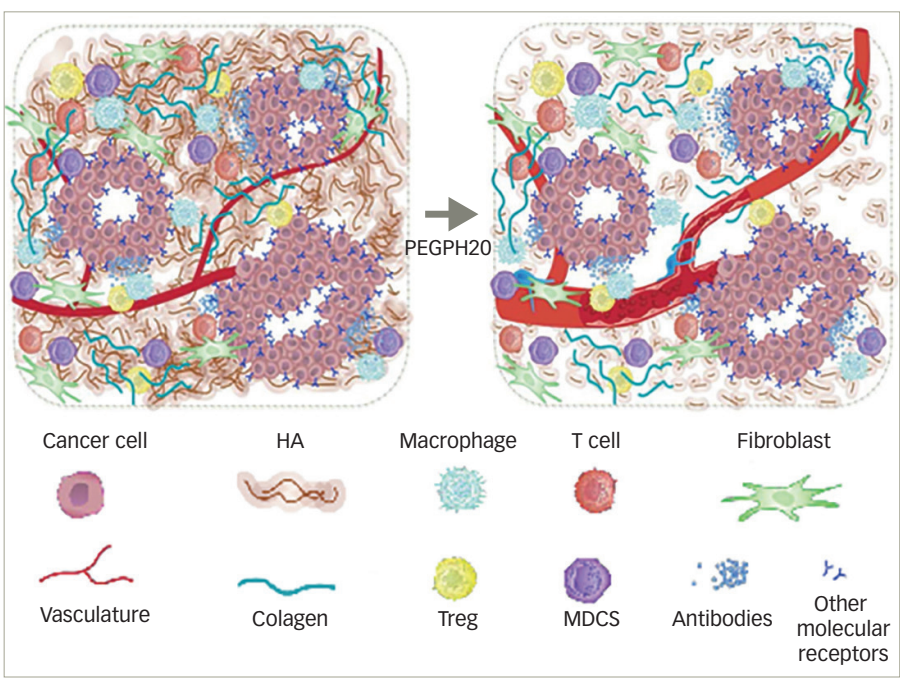

Removal of HA by PEGPH2O in HA-High tumor animal models was shown to decrease intratumoral pressure, decompress vasculature, increase perfusion, increase access for therapeutics, and increase access for immune cells. HA = hyaluronan; MDCs = myeloid dendritic cells; Tregs = regulatory T cells. Reproduced with permission from Halozyme. OHalozyme 2017.

include a dense extracellular matrix (ECM), fibroblasts, inflammatory cells, and abnormal vasculature. ${ }^{6}$ The TME can profoundly influence tumor behavior and response to therapy, and as a result it has emerged as an important target for therapy and as a major focus of cancer research. ${ }^{7}$ Within the TME, hyaluronan (HA) is a naturally occurring linear polysaccharide, and a major component of the tumor stroma in many solid tumors, including PDA. ${ }^{8}$ In animal models, accumulation of HA increases tumor interstitial fluid pressure (IFP), which in turn compresses blood vessels and compromises blood flow. ${ }^{9-12}$ This process leads to hypoxia in the tumor, which is a contributory factor in metastasis. In response to low oxygen levels, tumor cells trigger the synthesis of hypoxia-inducible factor 1a (HIF1a), a protein that directly represses the transcription of miR34a microRNA. Furthermore, this down-regulation of the microRNA is a prerequisite for hypoxia-induced epithelial-to-mesenchymal transition (EMT). In this process, HIF1a activates a genetic program that results in the transformation of non-invasive cells (which grow in a regulated fashion in epithelial sheets) into invasive, migratory cells that can develop new tumors elsewhere. ${ }^{13,14}$

Accumulation of HA in tumors may also act as a barrier to immune cells, including T lymphocytes and macrophages, creating an immune suppressed microenvironment. 15-17 Increased HA also prevents chemotherapeutic agents and monoclonal antibodies from reaching their sites of action. ${ }^{.9} \mathrm{HA}$ accumulation in the TME is associated with accelerated tumor growth and is an independent, negative predictor of survival. ${ }^{18-24}$

\section{PEGylated recombinant human hyaluronidase Mechanism of action}

A notable development in targeting HA in PDA has been the emergence of the novel investigational agent, PEGPH2O (pegvorhyaluronidase alfa), which is the PEGylated form of the recombinant human hyaluronidase, PH2O. PEGylation of this enzyme prolongs circulatory time to facilitate
HA degradation in the extracellular matrix surrounding tumor cells, enhancing consequent remodeling of the tumor stroma (see Figure 1).12

In animal models and in studies on various types of cancer, accumulation of HA has been shown to promote tumorigenesis. $8,912,25,26$ Conversely, HA depletion reverses these changes and inhibits tumor growth. However, the precise mechanism of PEGPH20-induced HA depletion is not completely understood. ${ }^{27}$ Preclinical studies show that treatment with PEGPH2O increases vascular perfusion in the tumor, which decreases or eliminates hypoxia and causes other alterations in the TME. ${ }^{8,12}$ Other in vivo studies in both animal and human tumors show that PEGPH20 decreases hypoxiarelated protein expression and induces translocation of proteins such as E-cadherin and $\beta$-catenin to the plasma membrane. ${ }^{8}$

Preclinical studies have also shown that PEGPH20-mediated HA reduction facilitates immune cell access to the TME, and increases CD8+ T cell recruitment to tumor sites. ${ }^{17,28}$ In animal models of PDA treated with PEGPH2O plus chemotherapy or monoclonal antibodies, the biophysical effects of HA degradation by PEGPH2O translate into reduced tumor growth and improved survival.9,10,12,29,30 In addition, adenosine, antibodies, and other molecular receptors are involved with dampening inflammation caused by HA degradation.

In patients with PDA and in other malignancies, levels of HA in tumors are variable, but tumors that accumulate HA (termed HA-High tumors) are more likely to respond to PEGPH2O treatment. In such tumors this treatment mediates HA reduction and this has been shown to decrease tumor hypoxia and consequently reduce metastases. ${ }^{31,32}$ In vitro and in vivo studies have shown that depletion of HA as a result of treatment with PEGPH2O leads to increased natural killer (NK) cell access to HAHigh tumor cells and enhanced trastuzumab or cetuximab-antibodydependent cell-mediated cytotoxicity. ${ }^{17}$ This further supports the belief that surrounding PDA tumors, the HA matrix forms a barrier that inhibits monoclonal antibody and NK cell access; this barrier can be overcome using PEGPH2O. In tumor cell membranes, CD44 receptors and receptors for hyaluronan mediated motility (RHAMM) bind and anchor the HA matrix, and this association is believed to enhance receptor tyrosine kinase activity that drives tumor progression and increases treatment resistance. ${ }^{27}$ Therefore, PEGPH2O may have some antitumor properties resulting from reduced signaling via CD44/RHAMM.

Overall, the preclinical and clinical evidence indicates that PEGPH2O has multiple antitumor effects but all are believed to result in substantial changes in the TME. These effects may inhibit progression, reduce tumor interstitial fluid pressure and improve vascular perfusion in HA-High tumors, resulting in increased access by immune cells, antibodies and anticancer therapies. ${ }^{910,1229}$

\section{Clinical studies}

Early studies of single-agent PEGPH2O in patients with metastatic cancer, including PDA, identified muscle spasms, arthralgia, and myalgia as dose-limiting; maximum tolerated dose was found to be $3 \mu \mathrm{g} / \mathrm{kg}$ twice weekly. ${ }^{33}$ In a subsequent phase $\mathrm{Ib}$ trial of PEGPH2O plus standard-dose gemcitabine in patients with previously untreated metastatic PDA, a recommended phase II dose for PEGPH2O $(3 \mu \mathrm{\mu g} / \mathrm{kg})$ was confirmed. ${ }^{34}$ Adverse events were again primarily musculoskeletal but did not lead to discontinuation. Exploratory imaging analyses in a subset of patients 
showed an increase in tumor perfusion at 24 hours, as assessed by dynamic contrast-enhanced magnetic resonance imaging (DCE-MRI), and a sustained reduction in tumor metabolic activity by fluorodeoxyglucose positron emission tomography with integrated computed tomography (F-FDG-PET/CT); these findings support the described mechanism of action of PEGPH2O that HA degradation improves access to the tumor site, thereby increasing exposure of systemically administered anticancer therapies. This study also demonstrated that patients with HA-High tumors might benefit most from PEGPH20-based treatment, a premise studied in a phase II trial and in an ongoing randomized, double-blind phase III trial.

More recent clinical findings that support PEGPH20 use in PDA come from a phase II, randomized, multicenter study in patients with stage IV, previously untreated disease (the HALO 109-202 study, NCT01839487; $n=279) .{ }^{34}$ Patients received PEGPH20 plus nab-paclitaxel and gemcitabine (PAG), or nab-paclitaxel and gemcitabine (AG) chemotherapy alone. In this study, HA was tested as a biomarker to potentially identify patients who may be more likely to benefit from PEGPH20 treatment. Tumor samples - formalin-fixed paraffin-embedded tissues from core samples - were analyzed using an affinity histochemical assay (VENTANA HA RXDX Assay), co-developed by Halozyme Therapeutics, Inc. and Ventana Medical Systems Inc. (Tucson, AZ, USA), to analyze levels of HA in solid tumors and potentially predict which patients might better respond to the addition of PEGPH2O in cancer treatments. ${ }^{35}$ This assay has been reported to have an inter-reader precision level of $94 \%$. $^{35}$

The HALO 109-202 study was conducted in two stages. Stage 1 data $(n=146)$ comprised the 'training set' for development of the VENTANA HA RXDX Assay and scoring algorithm. ${ }^{36}$ Patients with untreated metastatic PDA were randomized 1:1 to PAG twice weekly plus AG weekly, or AG once every 28 days. In this stage, approximately $40 \%$ of patients in the PAG arm during stage I of the trial developed a thromboembolism (TE), and as such were removed from study participation. The trial was consequently put on hold while the protocol was amended to include TE prophylaxis. TEs are a wellestablished complication of metastatic PDA and are further compounded by chemotherapy administration. As a result of this experience, in stage 2 patients at high risk of TE were excluded from the study and prophylactic enoxaparin was initiated. Under the revised protocol, stage 2 enrolled an additional 133 patients who were randomized 2:1 to PAG or AG treatment. The proportion of patients with HA-High tumors was 32\% in stage 1 and $28 \%$ in stage 2.

Data from stage 1 were used to select the $50 \%$ cut-off point for HA (i.e. the $50 \%$ HA level from the range of HA levels found in study patients' tumors). ${ }^{37}$ In this respect this study was novel in identifying a biomarker with the potential to guide patient selection. Combined data from stages 1 and 2 of the HALO 109-202 study presented at the Annual Meeting of the American Society for Clinical Oncology, 2017, ${ }^{38}$ show a robust signal for improved progression-free survival (PFS) when the cut-off for classifying patients as HA-High using the Ventana HA RXDX assay was set at $\geq 50 \%$. Using this criterion, of 246 patients with HA data, 84 were classified as HA-High. The primary endpoint of PFS was significantly higher for PAG versus AG treatment: 6.0 months versus 5.3 months, respectively; hazard ratio (HR) 0.73 ; $95 \%$ confidence interval (CI) 0.53-1.00; $p=0.048$. A PFS increase of 4 months in pancreatic cancer is a clinically relevant and substantial improvement. This result was even more striking in HA-High patients (9.2 months versus 5.2 months, respectively; HR $0.51 ; 95 \%$ Cl 0.26-1.00; $p=0.048){ }^{38}$
In the combined HALO 109-202 study analysis, TEs were primarily venous in nature but incidence was comparable between treatment arms (14\% for PAG versus $10 \%$ for $A G$ ) following enoxaparin initiation. Treatmentrelated adverse events of any grade associated with either PAG or AG treatment included peripheral edema (63\% for PAG versus $26 \%$ for AG), muscle spasms (56\% versus 3\%), neutropenia (34\% versus 19\%), and myalgia (26\% versus $7 \%)^{38}$

The HALO 109-202 study therefore met its key endpoints including improvement in the primary outcome of improved PFS in all patients receiving PAG compared with $A G$, and in the secondary endpoint of improved PFS in HA-High patients receiving PAG versus AG. Stage 2 of the HALO 109-202 study also met its primary safety endpoint of TE reduction. Positive trends in PFS and overall survival (OS) were observed in HA-High patients, supporting the ongoing phase III investigation of PAG versus AG in patients with HA-High PDA. ${ }^{38}$

To date, there have been no studies investigating PEGPH2O as monotherapy in PDA. All studies have investigated PEGPH2O used in combination with chemotherapy. PEGPH2O is currently under investigation in two small studies: one is investigating PEGPH2O for the treatment of patients with PDA without metastasis prior to resection surgery (NCT02241187), and one is investigating the treatment of patients with unresectable PDA (NCT02910882). Favorable findings in these trails and larger trials could increase indications in PDA suitable for PEGPH20 treatment.

\section{Perspective and future directions}

Pancreatic cancer is characterized by a profuse desmoplastic stroma, which is associated with aggressive pathogenesis and treatment resistance. ${ }^{6,39}$ This HA-rich stroma compresses tumor vasculature and provides a barrier to delivery of antineoplastic agents, including chemotherapeutics and therapetic antibodies, and immune cell infiltration. Overcoming this barrier offers an innovative strategy for improving anticancer therapy that may be applicable to multiple diseases at varying stages.

Previous efforts to target the PDA TME, however, have not been as successful. Specifically, clinical trials evaluating the roles of vascular endothelial growth factor inhibitors and the Hedgehog pathway inhibitor, IPI-926, have yielded disappointing results. ${ }^{40}$ Other approaches to potentially address the TME in PDA include treatment with heparin sulfate mimetic, M402 (NCT01621243), secreted protein rich in cysteine (SPARC) ${ }^{41}$ or evofosfamide; $; 2$ however, as yet, these approaches have not shown any notable evidence of efficacy. Targeting HA is a novel approach to address the vital role the microenvironment plays in PDA pathogenesis.

Currently, many therapies targeting PDA, TME and HA are under investigation (Table 1). ${ }^{43-46} \mathrm{HA}$ degradation by PEGPH2O is the most studied concept to date and is the most advanced in terms of development. Multiple preclinical and clinical studies have demonstrated that targeting the biophysical barrier to drug delivery in PDA with PEGPH2O is both feasible and promising. 12,25,34,37 Results from both the phase $\mathrm{Ib}^{34}$ and phase $\|(\mathrm{HALO}-202)^{38}$ clinical trials have demonstrated that the administration of PEGPH2O with different cytotoxic chemotherapy combinations is feasible and effective.

The results from the phase II HALO 109-202 study, in particular, demonstrated that the $\geq 50 \%$ HA cut-off limit can be used as a reliable biomarker to screen patients who may benefit from PEGPH20 treatment. ${ }^{38}$ This was a novel 
Table 1: Ongoing studies investigating efficacy/safety of PEGylated recombinant human hyaluronidase in cancer

\begin{tabular}{|c|c|c|c|c|c|}
\hline Study name/description & $\begin{array}{l}\text { ClinicalTrials.gov } \\
\text { identifier }\end{array}$ & $\begin{array}{l}\text { Phase intervention } \\
\text { model }\end{array}$ & Condition & Intervention & Primary endpoint \\
\hline $\begin{array}{l}\text { PEGPH20 plus nab-paclitaxel + gem versus } \\
\text { nab-paclitaxel + Gem in subjects with stage } \\
\text { IV untreated pancreatic cancer (study 202) }\end{array}$ & NCT01839487 & $\begin{array}{l}2 \text { parallel } \\
\text { assignment }\end{array}$ & Metastatic PDA & $\begin{array}{l}\text { PEGPH2O + nab-paclitaxel } \\
\text { + Gem versus nab- } \\
\text { paclitaxel + Gem }\end{array}$ & Progression-free survival \\
\hline $\begin{array}{l}\text { Phase II study: PEGPH2O with concurrent } \\
\text { Gem and Radiotherapy in patients with } \\
\text { localized, unresectable pancreatic } \\
\text { adenocarcinoma }\end{array}$ & NCT02910882 & $\begin{array}{l}2 \text { single group } \\
\text { assignment }\end{array}$ & $\begin{array}{l}\text { Non-resectable } \\
\text { pancreatic } \\
\text { adenocarcinoma }\end{array}$ & $\begin{array}{l}\text { PEGPH20 with concurrent } \\
\text { Gem + radiotherapy }\end{array}$ & $\begin{array}{l}\text { Number of participants with } \\
\text { treatment-related adverse events }\end{array}$ \\
\hline $\begin{array}{l}\text { A randomized, open-label, phase Ib/II study } \\
\text { of eribulin mesylate in combination with } \\
\text { PEGPH20 versus eribulin mesylate alone } \\
\text { in subjects with HER2-negative, High-HA } \\
\text { metastatic breast cancer }\end{array}$ & NCT02753595 & $\begin{array}{l}\text { 1b/2 parallel } \\
\text { assignment }\end{array}$ & $\begin{array}{l}\text { Metastatic breast } \\
\text { cancer }\end{array}$ & $\begin{array}{l}\text { Eribulin mesylate }+ \\
\text { PEGPH20 versus eribulin } \\
\text { mesylate alone }\end{array}$ & $\begin{array}{l}\text { Recommended phase } \| \text { dose }= \\
\text { objective response rate }\end{array}$ \\
\hline $\begin{array}{l}\text { Study of Gem, nab-paclitaxel, PEGPH20 and } \\
\text { rivaroxaban for advanced PDA }\end{array}$ & NCT02921022 & Parallel assignment & $\begin{array}{l}\text { Advanced/ } \\
\text { metastatic PDA }\end{array}$ & $\begin{array}{l}\text { Gem + nab-paclitaxel } \\
+ \text { PEGPH20 }\end{array}$ & Rate of symptomatic TE events \\
\hline $\begin{array}{l}\text { A phase III, randomized, double-blind, } \\
\text { placebo-controlled, study of PEGPH20 in } \\
\text { combination with nab-paclitaxel plus Gem } \\
\text { versus placebo plus nab-paclitaxel and } \\
\text { Gem in participants with HA-High stage IV } \\
\text { previously untreated PDA }\end{array}$ & NCT02715804 & $\begin{array}{l}3 \text { parallel } \\
\text { assignment }\end{array}$ & PDA & $\begin{array}{l}\text { PEGPH20 + nab-paclitaxel } \\
+ \text { Gem versus placebo + } \\
\text { nab-paclitaxel + Gem }\end{array}$ & $\begin{array}{l}\text { Progression-free survival + } \\
\text { overall survival }\end{array}$ \\
\hline $\begin{array}{l}\text { Perioperative stromal depletion strategies } \\
\text { in PDA }\end{array}$ & NCT02487277 & $\begin{array}{l}2 \text { single group } \\
\text { assignment }\end{array}$ & PDA & $\begin{array}{l}\text { PEGPH20 + nab-paclitaxel } \\
+ \text { Gem }\end{array}$ & $\begin{array}{l}\text { Clinically relevant pancreatic fistula; } \\
\text { pathologic complete response }\end{array}$ \\
\hline $\begin{array}{l}\text { A phase Ib open-label study of PEGPH } 20 \\
\text { with pembrolizumab in subjects with } \\
\text { selected hyaluronan high solid tumors }\end{array}$ & NCT02563548 & $\begin{array}{l}\text { 1b single group } \\
\text { assignment }\end{array}$ & $\begin{array}{l}\text { NSCLC; gastric } \\
\text { cancer }\end{array}$ & $\begin{array}{l}\text { PEGPH2O + } \\
\text { pembrolizumab }\end{array}$ & $\begin{array}{l}\text { Number of relapsed/refractory } \\
\text { NSCLC and relapsed/refractory } \\
\text { gastric adenocarcinoma subjects } \\
\text { experiencing dose-limiting toxicities } \\
\text { during cycle } 1 \text { (day } 1 \text { through day 21) }\end{array}$ \\
\hline
\end{tabular}

Gem = gemcitabine; PDA = pancreatic ductal adenocarcinoma; HER2 = human epidermal growth factor receptor 2 PEGPH2O = PEGylated recombinant human hyaluronidase; $N S C L C=$ non-Small cell lung cancer; $T E=$ symptomatic thromboembolic.

aspect of the study and showed that the level of HA in patients receiving PEGPH2O is a critical factor, and is one of the only predictive biomarkers currently under prospective evaluation in PDA. The HALO 109-202 study also demonstrated improved efficacy of PEGPH20 in combination with AG compared with AG alone in terms of PFS and secondary endpoints (PFS by HA level, overall response rate and overall survival), especially for HA-High patients. These exciting findings justify and support the ongoing phase III HALO 109-301 study (NCT02715804), in which a larger patient population $(n=420)$, all of whom have HA-High tumors (using the same $\geq 50 \%$ HA cutoff point), are randomized to treatment with PAG or AG. This pivotal study incorporates PFS and OS as co-primary endpoints and will provide key data on the utility of PEGPH2O in metastatic PDA treatment.

The risk of TE that emerged in the HALO 109-202 study has been shown to be mitigated when PEGPH2O is given concurrently with prophylactic low molecular weight heparin anticoagulation therapy. Additional toxicities attributed to PEGPH2O include edema, neutropenia, and musculoskeletal effects (MSKEs; myalgia and muscle spasms). The musculoskeletal effects associated with this medication are quite unique. Patient-reported muscle cramping is an uncommon drug-related toxicity with few standard treatment approaches. MSKES associated with PEGPH2O have been found to be quite manageable and are infrequently dose limiting or a reason for drug discontinuation. Anti-inflammatory medications, as well as cramping emollients, are both quite helpful, and MSKEs are reduced with use of corticosteroids (dexamethasone). In addition, patients may benefit from concurrent muscle relaxant, non-steroidal anti-inflammatory drugs (NSAID), or topical agents, as needed. In rare cases, temporary courses of opiate pain medications are needed for adequate pain control.

Overcoming therapeutic resistance through stroma-targeting agents, like PEGPH20, may provide an ideal complement to multiagent chemotherapy. Already, PEGPH2O is being studied in combination with one of the current standards of care for first-line chemotherapy, which is currently used to treat metastatic PDA: nab-paclitaxel/gemcitabine. However, recruitment to a SWOG (formerly Southwest Oncology Group) phase Ib/ II trial investigating the addition of PEGPH2O to FOLFIRINOX in patients with untreated PDA has been permanently closed to enrolment due to a predicted, unlikely statistically significant, advantage in OS. ${ }^{47}$ Despite this setback, we envision a future where PEGPH2O may be combined with, and improve efficacy of, other antineoplastic regimens to treat both earlystage and metastatic PDA.

The development of PEGPH2O as a stromal targeting agent, and the identification of a subgroup of patients more likely to benefit from it, is changing the treatment paradigm of PDA. The VENTANA HA RXDX Assay for selection of patients with HA-High tumors is one of the only potentially predictive biomarkers for PDA under prospective evaluation. This biomarker will allow us to stratify PDA patients into groups most likely to benefit from 
PEGPH2O-mediated HA degradation and allow HA-low patients to pursue alternative approaches. HA-degradation by PEGPH2O is also being studied in other tumor types, including breast, gastric and non-small cell lung cancers (NSCLC), and will soon be studied in combination regimens for other HA-High cancers.

Preclinical studies have also demonstrated that the malignant stroma that is rich in HA restricts tumor immune cell and antibody infiltration. The combination of PEGPH2O and pembrolizumab, a humanized monoclonal antibody that targets programmed cell death receptor 1 (PD1), is currently being studied in patients with cancers such as NSCLC and gastric cancers (NCT02563548). Overcoming the stromal barrier may extend the benefits of
PD1 blockade to populations whose disease has been refractory to single agent checkpoint inhibitors, as we have seen in PDA and other diseases.

Advances in the understanding of the PDA TME and the development of agents targeting HA are rapidly changing the PDA treatment landscape. Targeting the stroma, overcoming treatment resistance, and stratification of patients are providing a new framework for more successful treatment of this disease. This is allowing the barrier to effective PDA treatments to be broken, and provides a shift away from predominantly poor prognosis that oncologists have confronted hitherto. These new approaches have the potential to provide new hope and direction for the pancreatic cancer community. $\square$
1. Hidalgo $\mathrm{M}$, Cascinu S, Kleeff J, et al., Addressing the challenges of pancreatic cancer future directions for improving outcomes, Pancreatology, 2015;15:8-18.

2. Siegel RL, Miller KD, Jemal A, Cancer Statistics, 2017, CA Cancer J Clin, 2017:67:7-30.

3. Hirshberg Foundation for Pancreatic Cancer Research, Pancreatic Cancer Facts, 2017, http://pancreatic.org/pancreatic-cancer/ pancreatic-cancer-facts/ (accessed November 3, 2017).

4. Rahib L, Smith BD, Aizenberg R, et al., Projecting cancer incidence and deaths to 2030: the unexpected burden of thyroid, liver, and pancreas cancers in the United States, Cancer Res, 2014;74:2913-21

5. Stromnes IM, DelGiorno KE, Greenberg PD, et al., Stroma reengineering to treat pancreas cancer, Carcinogenesis, 2014:35:1451-60.

6. Rasheed ZA, Matsui W, Maitra A, Pathology of pancreatic stroma in PDAC. In: Grippo PJ and Munshi HG (eds.), Pancreatic Cancer and Tumor Microenvironment, Trivandrum (India): Transworld Research Network, 2012

7. Martin M, Wei H, Lu T, Targeting microenvironment in cancer therapeutics, Oncotarget, 2016:7:52575-83.

8. Kultti A, Zhao C, Singha NC, et al., Accumulation of extracellular hyaluronan by hyaluronan synthase 3 promotes tumor growth and modulates the pancreatic cancer microenvironment, Biomed Res Int, 2014;2014:817613.

9. Jacobetz MA, Chan DS, Neesse A, et al., Hyaluronan impairs vascular function and drug delivery in a mouse model of pancreatic cancer, Gut, 2013:62:112-20.

10. Provenzano PP, Cuevas $C$, Chang $A E$, et al., Enzymatic targeting of the stroma ablates physical barriers to treatment of pancreatic ductal adenocarcinoma, Cancer Cell, 2012:21:418-29.

11. Stylianopoulos T, Martin JD, Snuderl M, et al., Coevolution of solid stress and interstitial fluid pressure in tumors during progression: implications for vascular collapse, Cancer Res, 2013;73:3833-41.

12. Thompson CB, Shepard HM, O'Connor PM, et al., Enzymatic depletion of tumor hyaluronan induces antitumor responses in depletinical animal models, Mol Cancer Ther, 2010:9-3052-64.

13. Gilkes DM, Semenza GL, Wirtz D, Hypoxia and the extracellular matrix: drivers of tumour metastasis, Nat Rev Cancer, 2014;14:430-9

14. Li H, Rokavec $M$, Jiang $L$, et al., Antagonistic Effects of $p 53$ and HIF1A on microRNA-34a Regulation of PPP1R11 and STAT3 and Hypoxia-induced Epithelial to Mesenchymal Transition in Colorectal Cancer Cells, Gastroenterology, 2017:153:505-20.

15. Clift R, Cowell JA, Zimmerman S, et al., PEGylated recombinant hyaluronidase $\mathrm{PH} 20$ (PEGPH2O) enhances pemetrexed antitumo efficecy on a human nonsquamous NSCLC xenograft model, Presented at: 107th Annual Meeting of the American Association for Cancer Research, New Orleans, LA, USA, 16-20 April 2016. Abstr 283.

16. Oberstein $\mathrm{PE}$, Olive KP, Pancreatic cancer: why is it so hard to treat? Therap Adv Gastroenterol, 2013;6:321-37.

17 Singha NC, Nekoroski T, Zhao $C$, et al. Tumor-associated hyaluronan limits efficacy of monoclonal antibody therapy, $\mathrm{MOl}$ Cancer Ther, 2015;14:523-32.
18. Anttila MA, Tammi RH, Tammi MI, et al., High levels of stromal hyaluronan predict poor disease outcome in epithelial ovarian cancer, Cancer Res, 2000:60:150-5.

19. Auvinen P, Tammi R, Parkkinen J, et al., Hyaluronan in peritumora stroma and malignant cells associates with breast cancer spreading and predicts survival, Am J Pathol, 2000:156:529-36.

20. Lipponen P, Aaltomaa S, Tammi R, et al., High stromal hyaluronan level is associated with poor differentiation and metastasis in prostate cancer, Eur J Cancer, 2001;37:849-56.

21. Pirinen R, Tammi R, Tammi M, et al., Prognostic value of hyaluronan expression in non-small-cell lung cancer. Increased stromal expression indicates unfavorable outcome in patients with adenocarcinoma, Int I Cancer 2001:95:12-7.

22. Ropponen $\mathrm{K}$, Tammi M, Parkkinen J, et al., Tumor cell-associated hyaluronan as an unfavorable prognostic factor in colorectal cancer, Cancer Res, 1998;58:342-7.

23. Setala LP, Tammi MI, Tammi RH, et al., Hyaluronan expression in gastric cancer cells is associated with local and nodal spread and reduced survival rate, Br J Cancer, 1999:79:1133-8.

24. Whatcott $\mathrm{CJ}$, Diep $\mathrm{CH}$, Jiang $\mathrm{P}$, et al., Desmoplasia in primary tumors and metastatic lesions of pancreatic cancer, Clin Cancer Res, 2015:21:3561-8.

25. Jiang P, Li X, Thompson CB, et al., Effective targeting of the tumor microenvironment for cancer therapy, Anticancer Res, 2012;32:1203-12

26. Tammi RH, Kultti A, Kosma VM, et al., Hyaluronan in human tumors: pathobiological and prognostic messages from cell-associated and stromal hyaluronan, Semin Cancer Biol, 2008:18:288-95.

27. Shepard $\mathrm{HM}$, Breaching the castle walls: Hyaluronan depletion as therapeutic approach to cancer therapy, Front Oncol, 2015;5:192.

28. Lee J, Leong M, Huang Y, et al., Combination of PEGylated recombinant hyaluronidase PH2O (PEGPH20) with live-attenuated, double-deleted (LADD) Listeria enhances tumor infiltrating CD8+ T cell response and antitumor efficacy in mice LB-198, Presented at: American Association for Cancer Research, Washington DC USA, 1-5 April 2017. Abstr LB-198.

29. Osgood RJ, Skipper JF, Cowell JA, et al., PEGylated recombinan human hyaluronidase PH2O (PEGPH20) enhances nab-paclitaxel plus gemcitabine efficacy in human pancreatic cancer xenografts, Presented at: American Association for Cancer Research (AACR) Special Conference on Pancreatic Cancer, New Orleans, LA, USA 18-21 May 2014, Poster B86.

30. Rosengren S, Clift R, Zimmerman SJ, et al., PEGylated recombinant hyaluronidase $\mathrm{PH} 2 \mathrm{O}$ ( $\mathrm{PEGPH} 20$ ) enhances checkpoint inhibitor efficacy in syngeneic mouse models of cancer, Presented at: 107th Annual Meeting American Association for Cancer Research, New Orleans, LA, USA, 16-20 April 2016. Abstract 4886.

31. Cowell J, Zimmerman SJ, Marrela M, et al., PEGPH2O increases the anticancer activity of standard chemotherapy combinations, vincristine (VIN) and D actinomycin (DACT), in a Wilms' xenograft model, Presented at: 107th Annual Meeting of the American Association for Cancer Research, New Orleans, LA, USA, 16-20 April 2016. Abstract 2463

32. LiX, Jiang P, Symons R, et al., PEGylated human recombinant hyaluronidase $\mathrm{PH} 2 \mathrm{O}$ reduces solid tumor hypoxia, Presented at: 103rd Annual Meeting of the American Association for Cancer Research, 31 March-4 April 2012, Chicago, IL, USA. Abstract 3796.

33. Infante, British Journal of Cancer (submitted), 2017;

34. Hingorani SR, Harris WP Beck JT, et al . Phase Ib study of PEGylated recombinant human hyaluronidase and gemcitabine in patients with advanced pancreatic cancer, Clin Cancer Res, 2016;22:2848-54.

35. Pu J, Aldrich C, Zhu J, et al., Hyaluronan assessment in tumor microenvironment using new affinity histochemistry assay and scoring method, J Clin Oncol, 2017;35(suppl; abstr e23196).

36. Halozyme, Study 202 overall results and stage 2 results, 2017 https://s21.q4cdn.com/250105458/files/doc presentations/2017/ Halozyme-January-2017-Investor-Presentation Final.pdf (accessed November 3,2017 ).

37. Bullock AJ, Hingorani SR, Wu XW, et al., Final analysis of stage 1 data from a randomized phase II study of PEGPH20 plus nabPaclitaxel/gemcitabine in stage IV previously untreated pancreatic cancer patients (pts), utilizing Ventana companion diagnostic assay, J Clin Oncol, 2016;34(suppl; abstr 4104).

38. Hingorani $S$, Bullock A, Seery T, et al., Randomized phase 2 study of PEGPH2O plus nab-paclitaxel/gemcitabine (PAG) VS AG in patients (Pts) with untreated, metastatic pancreatic ductal adenocarcinoma (mPDA), Presented at: Annual Meeting of the American Society for Clinical Oncology, Chicago, IL, USA, 1-5 June 2017. Abstr 4008

39. Teague A, Lim KH, Wang-Gillam A, Advanced pancreatic adenocarcinoma: a review of current treatment strategies and developing therapies, Ther Adv Med Oncol, 2015; 7:68-84.

40. Olive KP, Jacobetz MA, Davidson CJ, et al., Inhibition of Hedgehog signaling enhances delivery of chemotherapy in a mouse model of pancreatic cancer Science, 2009:324:1457-61.

41. Vaz J, Ansari D, Sasor A, et al., SPARC: A potential prognostic and therapeutic target in pancreatic cancer, Pancreas, 2015;44:1024-35

42. Pourmorteza M, Rahman ZU, Young M, Evofosfamide, a new horizon in the treatment of pancreatic cancer, Anticancer Drugs,

43. Kohi S, Sato N, Koga A, et al., Hyaluromycin, a novel hyaluronidase inhibitor, attenuates pancreatic cancer cell migration and proliferation, J Oncol, 2016;2016:9063087.

44. Kudo D, Suto A, Hakamada K, The Development of a novel therapeutic strategy to target hyaluronan in the extracellular matrix of pancreatic ductal adenocarcinoma, Int J Mol SCi, 2017;18:pii:E600.

45. Nagase H, Kudo D, Suto A, et al., 4-Methylumbelliferone suppresses hyaluronan synthesis and tumor progression in SCID mice intra-abdominally inoculated with pancreatic cancer cells, Pancreas, 2017;46:190-7.

46. Sato $\mathrm{N}$, Cheng XB, Kohi S, et al., Targeting hyaluronan for the treatment of pancreatic ductal adenocarcinoma, Acta Pharm $\sin B, 2016 ; 6: 101-5$.

47. Halozyme, Inc, Halozyme Provides Update On SWOG Collaborative Group Clinical Study, 2017, www.halozyme.com/investors/ news-releases/news-release-details/2017/Halozyme-ProvidesUpdate-On-SWOG-Collaborative-Group-Clinical-Study/default. aspx\#sthash.2yBjf1Mh.dpuf (accessed November 8, 2017). 